\title{
Genomic Profiling in ctDNA Revealing Complicated Resistance Mechanism of Olaparib and Abiraterone as Salvage Therapy in Prostate Cancer: A Case Report
}

\author{
Fang Yuan \\ Chongqing University \\ Nan Liu \\ Chongqing University \\ Hong Luo \\ Chongqing University \\ XiaoTian Zhang \\ BGI \\ MingZhen Yang \\ Army Medical University \\ Hong Zhou (D zhouhongcqch@126.com) \\ Chongqing University https://orcid.org/0000-0003-2526-8153
}

\section{Case Report}

Keywords: mCRPC, Olaparib, PALB2

Posted Date: July 9th, 2020

DOI: https://doi.org/10.21203/rs.3.rs-38627/v1

License: (a) (i) This work is licensed under a Creative Commons Attribution 4.0 International License.

Read Full License 


\section{Abstract}

Background: PARP inhibitor, e.g. Olaparib, displayed superior clinical effect in metastatic castration prostate cancer (MCRPC) patients with deleterious mutations of DNA damage repair genes (DDR) as reported recently. Besides, for $\mathrm{MCRPC}$ patients without DDR alterations, a combination of Olaparib and abiraterone may achieve an acceptable clinical outcome as indicated by researches. However, these previous clinical studies involved patients strictly following inclusion criteria. In real-world situations, where the situation of patients is more complicated, the efficacy of salvage treatment of Olaparib with or without abiraterone-prednisone remains to be unclear.

Case presentation: The present case displayed a 61-year-old man who was initially diagnosed with metastatic hormone-sensitive prostate cancer(mHSPC) and proceeding into mCRPC after several kinds of standard therapies. Surprisingly, the patient had a well TPSA response and remission of symptoms for four months by taking Olaparib combined with abiraterone-prednisone, basing on androgen-deprivation therapy (ADT). The resistance of Olaparib was occurred with slowly increasing serum TPSA level again.

Conclusion: Resistance mechanism as discovered by comprehensive genomics profiling. The major concern was that two somatic reversion mutations occurred in PALB2 recovering its reading framer storing the function of the primary PALB2 mutation and caused the resistance to a PARP inhibitor.

\section{Background}

Prostate cancer is sixth mostly occurred malignant tumor in the mainland China[1]. Patients with metastatic castration prostate cancer (MCRPC), as the most dangerous subtype, often have an unfavored clinical outcome as well as poor life quality[2]. Therapeutic options when progressing to MCRPC are limited besides the administration of second-generation androgen receptor inhibitor, abiraterone or docetaxel together with prednisone, based on androgen-deprivation therapy (ADT).Poly (ADP-ribose) polymerase (PARP) inhibitor displayed promising clinical performance and prolonged survival duration, life quality in HRR gene mutated patients with ovarian cancer[3], breast cancer[4], pancreatic cancer[5] and prostate cancer[6, 7]. Recent studies showed potential clinical efficacy of Olaparib in mCRPC patients, especially carrying deleterious mutations of DNA damage repair (DDR) genes. Among these genes, BRCA2 deleterious mutation may serve as an appropriate indicator for the response to PARP inhibitor. Lately released results from PROfound study[8] also indicated a promising clinical outcome when patients with $B R C A 1 / 2$ or $A T M$ deleterious mutations receiving Olaparib treatment. Meanwhile, for patients without DDR deleterious mutations, a combination of Olaparib together with abirateroneprednisone may be used as an optimal therapeutic approach[9]. However, clinical trials involved patients strictly following inclusion criteria. Therefore, in a real-world situations, where the status of patients is more complicated, the efficacy of salvage treatment of Olaparib with or without abiraterone mains to be unclear.

\section{Case Presentation}


A 61-year-old man went to our hospital due to continuous lumbosacral pain for three months. The magnetic resonance imaging (MRI) of the lumbar vertebrates showed multiple bone space-occupying lesions (Fig. 1a). Bone scanning further displayed the high metabolic activity of these lesions (Fig. 1b). MRI of the prostate indicated invasion of the seminal vesicle, bladder wall, and metastasis to pelvic lymph node (Fig. 1C). No special aberration was found in the lung. Together with a significantly increased level of serum total prostate-specific antigen (TPSA, $787 \mathrm{ng} / \mathrm{ml}$ ) and normal level of neuron-specific enolase (NSE, $4.32 \mathrm{ng} / \mathrm{ml}$ ), an ultra-sound guided transperineal prostate needle biopsy (altogether 14 needles) was conducted resulting in confirmed diagnosis of prostate adenocarcinoma with the highest Gleason score $(5+5=10)$ and extreme positive rate $(14 / 14)$. Immunohistochemistry $(\mathrm{IHC})$ staining on serial sections of biopsy tissue excluded neuro-endocrine component [CK-L(+), PSA(+), P504S(+), NKX3.1(+), AR(++) > 95\%, P63(-), CK34ßE12(-), Syn(-), CgA(-), CD56(-), ki-67 30\%(+)] (Fig. 1d). Thus, this patient was definitely and initially diagnosed with metastatic hormone-sensitive prostate cancer (mHSPC, pT4N1M1b, stage IV).

797Therefore, ADT in combination with docetaxel was applied as initial treatment[10-12], especially for mHSPC with high tumor burden[13]. Two cycles of therapeutic intervention significantly relieved patientreported pain as well as TPSA level dropped to $0.45 \mathrm{ng} / \mathrm{ml}$ (Fig. 2b). Due to severe bone marrow inhibition and liver toxicity, docetaxel was no longer administrated, but continuing androgen-deprivation therapy. Lumbosacral pain manifestation along with a bounce of increased TPSA was observed after 5 months, meanwhile testosterone levels remained castrated throughout, which indicated his disease progressed to mCRPC. To determine primary resistance and sensitivity for new endocrine therapy, we tested the number and classification of circulating tumor cells (CTCs) together with AR-V7 mRNA in these CTCs[14] (Method see Additional file 1). Based on the negative findings on the detection, we confidently decided to choose the abiraterone-prednisone administration for his maintenance therapy (Fig. 2a). Decreased TPSA level $(3.02 \mathrm{ng} / \mathrm{ml})$ was measured at the 6th month of treatment. Fortunately, the disease was effectively controlled by abiraterone for nearly one year. Elevated TPSA level $(69.57 \mathrm{ng} / \mathrm{ml})$ indicated the second time of disease progression. Increased number of CTCs along with overexpression of AR-V7 mRNA further confirmed abiraterone resistance (Fig. 2a-b). The patient was eligible for a phase III multicenter, randomized, double-blind, placebo-controlled clinical trial to evaluate the efficacy and safety of Proxalutamide (an AR antagonist) in patients with metastatic castration-resistant prostate cancer ( $\mathrm{mCRPC}$ ) who failed abiraterone acetate and docetaxel (The trial is not over yet). Unfortunately, the disease progressed rapidly, reflected in the increased TPSA level $(601 \mathrm{ng} / \mathrm{ml})$ along with severe bilateral lower limb edema and the presence of somnolence. Due to no germline BRCA1/2 deleterious mutation detected from his peripheral blood mononuclear cell at the first time of genetic testing, the tumor was rechallenged by abiraterone together with a PARP inhibitor, Olaparib. TPSA decreased rapidly and amazingly to $119.65 \mathrm{ng} / \mathrm{ml}$ from $601.61 \mathrm{ng} / \mathrm{ml}$ after one month's combined treatment and maintained at a very low level $(8.02 \mathrm{ng} / \mathrm{ml})$ (Fig. 2c). ECOG performance and mental state were significantly improved with decreased painful feelings as well as lowering morphine dosage. After five months of' clinical response to this combinational therapeutic regimen, TPSA climbed with increasing patient-reported carcinomatous pain and decreasing ECOG performance. When the disease progressed again, 
comprehensive genomic profiling for his ctDNA was performed. 508 cancer-related genes were sequenced by next-generation sequencing (Method see Additional file 1). PALB2 germline pathogenic mutation was unclosed. Meanwhile, 12 somatic variants as well as 3 copy number alterations were detected in following genes (PTEN, AR, CHD1, TP53, FGFR1, NOTCH2, PIK3C2G, LHCGR, CDC25C, FLT4) and especially two reverse missense mutations in $P A L B 2$ which may recover truncated poly-peptide chain translated from germline PALB2 mutation (Fig. 3, Table 1). In detail, two somatic mutations of PALB2, c.751_752delCAinsTT and c.751_753delCAGinsTAC (Fig. 3), shared same loci with the germline deleterious variant c.751C $>$ T. As a result, missense mutations led by these two in-frame deletion or insertion variants contributed to restore entire PALB2 reading frame and to make its function correctly in repairing DNA double-stranded breaks by homologous recombination repair pathway. Besides these two reversed mutations of PALB2, ctDNA profiling discovered a complicated resistance mechanism to the combinational therapy of Olaparib and abiraterone in this patient via activation of multiple signaling pathways.

Table 1

Summary of genomic alterations in ctDNA detected from the patient with resistance of Olaparib.

\begin{tabular}{|c|c|c|c|c|c|}
\hline Gene & Base change & $\begin{array}{l}\text { Amino acid } \\
\text { variation }\end{array}$ & Exon & $\begin{array}{l}\text { Variant } \\
\text { frequency }\end{array}$ & Transcript \\
\hline PTEN & c.136_137del & p.Y46Qfs*5 & EX2 & $59.47 \%$ & NM_000314.4 \\
\hline$A R$ & Copy number gain & & & & \\
\hline CHD1 & Copy number loss & & & & \\
\hline FGFR1 & Copy number gain & & & & \\
\hline TP53 & c.665_672*11del & - & $\begin{array}{l}\text { EX6- } \\
\text { IVS6 }\end{array}$ & $36.13 \%$ & NM_000546.5 \\
\hline $\mathrm{NOTCH} 2$ & c. $5311-1 \mathrm{G} \square \mathrm{A}$ & - & IVS29 & $1.28 \%$ & NM_024408.3 \\
\hline PIK3C2G & c. $2143 \mathrm{~A} \square \mathrm{G}$ & p.R715G & EX15 & $40.23 \%$ & NM_004570.4 \\
\hline LHCGR & c. $143 \mathrm{C} \square \mathrm{T}$ & p.T48M & EX1 & $23.39 \%$ & NM_000233.3 \\
\hline PALB2 & c.751_752delCAinsTT & p.Q251L & EX4 & $7.31 \%$ & NM_024675.3 \\
\hline PALB2 & c.751_753delCAGinsTAC & p.Q251Y & EX4 & $3.79 \%$ & NM_024675.3 \\
\hline PALB2 & c.751C口T & p.Q251* & EX4 & germline & NM_024675.3 \\
\hline$C D C 25 C$ & c.1150_1151delGGinsCC & p.G384P & EX12 & $2.35 \%$ & NM_001790.3 \\
\hline FLT4 & c.376G $\square \mathrm{A}$ & p.A126T & EX3 & $1.15 \%$ & NM_002020.4 \\
\hline
\end{tabular}

Indeed, functional assays are needed to validate resistant mechanisms indicated by these ctDNA findings. 


\section{Discussion}

Patients were considered to be short-lived when developed to mCRPC, despite significant progress in systemic and anti-androgen therapy. In this case, a mHSPC patient received multiple lines of therapy, and an acceptable clinical response to Olaparib and abiraterone-prednisone combination therapy was achieved. Genetic testing of ctDNA indicated a complicated mechanism for his final disease progression.

Previous studies reported the vital contribution of the alternative splice of AR-V7 to resistance to antiandrogen therapy $[15,16]$. In our case, when developed to mCRPC, CTCs and AR-V7 mRNA testing were negative. This patient had a nearly one-year progression-free interval receiving abiraterone mono-therapy, basing on testosterone levels that remained castrated throughout the entire process. Disease progression was mainly due to a secondary AR-V7 overexpression as well as the epithelial-mesenchymal transition of prostate cells as in parallel with reported investigations[17]. In addition, androgen receptor amplification or gain of function mutations of AR, as observed in ctDNA of this patient, would also continuously activate downstream AR signaling pathway overcoming extrinsic androgen inhibition $[18,19]$.

Recent progress delineated a subgroup of prostate patients, especially the loss of function mutations of BRCA2 gene, benefiting from the treatment of PARP inhibitors. In TOPARP-A and TOPARP-B study [6, 7], Olaparib mono-therapy has antitumor activity against $\mathrm{MCPRC}$ with HRR or DDR gene alterations. TOPARP-B study showed BRCA1/2 mutated mCRPC patients yielded an objective response rate of $83.3 \%$. Recently released results from PROfound study showed that Olaparib provided a statistically significant and clinically meaningful improvement on BICR assessed rPFS, especially for BRCA2-mutated patients[8]. A similar benefit was observed in TRITON2 (rucaparib)[20, 21] and GALAHAD (niraparib) study[22].

Neither germline BRCA2 nor BRCA1 deleterious mutation was found in this patient as shown by the first genetic testing. Supported by the conclusion from study 08[23], Olaparibin combination with abiraterone showed significant clinical benefit and this efficacy lasted for nearly five months. Progression was inevitably occurred, which led to the second time of genetic testing. Comprehensive genomic profiling uncovered that, instead of $B R B A 1 / 2$, this patient had a germline $P A L B 2$ nonsense mutation which may lead to truncation of PALB2 protein. As this patient was resistant to prior abiraterone treatment together with AR amplification and AR-V7 overexpression, abiraterone may not be able to block AR signaling triggering "BRCAness"[24]. PALB2, as one of tumor suppressor genes, by physically interacting with BRCA2 followed by recruiting RAD51 to DNA breaks, plays critical roles in repairing DNA double-strand breaks by homologous recombination (HR)[25]. A couple of studies indicated PALB2 deleterious mutations were associated with clinical benefit from PARP inhibitors[6, 20]. For instance, TOPARP-B study showed patients with PALB2 germline or somatic loss of function mutation may benefit from Olapraib treatment[6].

This patient had disease worsened after five months' disease relieving period, which indicated PARP inhibitor resistance. The most known mechanism is the secondary somatic reverse mutation in germline mutated genes. Goodall et al reported in a mCRPC patient with germline PALB2 p.L253lfs 2 , who had a 9month clinical benefit from Olaparib and two reverse somatic mutations in PALB2 were detected in his 
ctDNA at disease progression[26]. In our case, this patient had a germline PALB2 p.Q251* nonsense mutation. Two somatic reverse mutations which may restore $P A L B 2$ reading frame were observed when the disease progressed.

Besides reverse mutation as a potential mechanism on PARP inhibitor resistance, EwaGogola et al[27] concluded four major mechanisms of PARP inhibitor resistance including upregulation of drug efflux, restoration of homologous recombination, target-specific resistance to PARP inhibitor and restoration of stalled replication fork protection. Meanwhile, by the genomic profiling, we proposed restoration of homologous recombination was not only induced by reverse mutations of PALB2. Loss of function mutation of PTEN together with FGFR1 amplification may directly and indirectly activated PI3K-AKTmTOR as well as RAS-RAF-MAPK/ERK signaling pathway[28]. Activation of these oncogenic pathways may further drive the expression of HR genes compensating DNA double breaks. In addition, activation of these signaling pathway may accelerate cell-cycle and evade apoptosis[29]. In this case, the mechanism of PARP inhibitor resistance was explored in detail via comprehensive genomic profiling. Further validation at the functional level is warranted.

Several therapeutic issues need to be concerned. Firstly, as fully respecting patient's willingness, comprehensive genomic profiling can only be performed when the resistance of Olaparib occurred. In this circumstance, it could not distinguish the primary or secondary source of these two somatic PALB2 mutations. Secondly, therapeutically, instant multiple gene testing is definitely essential. Detection of germline PALB2 aberration at a relatively early stage may make this patient receive Olaparib monotherapy, but not being enrolled into the Proxalutamide clinical trial. Thirdly, PALB2 is a cancer susceptibility gene that may be increasing the risk of breast cancer as well as be substantially associated with the onset of ovarian cancer, pancreatic cancer and male breast cancer[30]. Concerning this patient carried a PALB2 germline pathogenic variation, his first and secondary degree relatives need to visit a genetic counselor for further evaluation.

\section{Conclusion}

Here we described a complete treatment period of a prostate cancer patient from mHSPC to mCRPC till death because of disease progression. Endocrine therapy, chemotherapy, and the target therapy utilizing PARP inhibitor as well as abiraterone were chosen. The prostate cancer with HRR gene mutation may be more sensitive to the PARP inhibitor, but we should also realize the occurrence of the resistance to the PARP inhibitor, such as the somatic reverse mutation. We also recommend that timely multi-gene testing is of great importance for patients, especially $\mathrm{MCRPC}$, to select a precise therapeutic approach.

\section{List Of Abbreviations}

mCRPC: metastatic castration prostate cancer; DDR: DNA damage repair; mHSPC: metastatic hormonesensitive prostate cancer; ADT: androgen-deprivation therapy; PARP: Poly (ADP-ribose) polymerase. 


\section{Declarations}

\section{Ethics approval and consent to participate}

Acquisition of tissue specimens and genomic analyses were approved by the Ethics Committee of Chongqing University Cancer Hospital,Chongqing,China.

\section{Consent for publication}

Written informed consent was obtained from the patient and his wife for the publication of this case report and any accompanying images. A copy of the written consent is available for review by the Editorin-Chief of this journal.

\section{Availability of data and materials}

The dataset supporting the conclusions of this article is included with in the article and its additional file 1.

\section{Competing interests}

The authors declare that they have no competing interests

\section{Funding}

This work was supported by the Major Project of Chongqing Health Committee (No.2016zdxm031), the Natural Science Foundation of Chongqing (No.cstc2018jcyjAX0781) and National Natural Science Foundation of China (No.81302316, No.81702452).

\section{Authors' contributions}

Fang Yuan and Nan Liu contributed equally as co-first authors in collecting clinical data and writing the paper; Fang Yuan and Hong Luo provided clinical treatment; Xiaotian Zhang performed the molecular genetic studies; Mingzhen Yang and Hong Zhou designed and coordinated the study and participated in draft preparation. The authors read and approved the final manuscript.

\section{Acknowledgments}

We thank lecturer Tarun Sarkar (School of Foreign Languages and Cultures, Chongqing University, Chongqing, China) for helping to polish the language.

\section{References}

1. Zheng RS, Sun KX, Zhang SW, Zeng HM, Zou XN, Chen R, et al. Report of cancer epidemiology in China, 2015. Zhonghua Zhong Liu Za Zhi. 2019;41(1):19-28. 
2. Robinson D, Van Allen EM, Wu YM, Schultz N, Lonigro RJ, Mosquera JM, et al. Integrative clinical genomics of advanced prostate cancer. Cell. 2015;161(5):1215-28.

3. Friedlander M, Moore KN, Colombo N, Scambia G, Kim B-G, Oaknin A, et al. Efficacy of maintenance olaparib for newly diagnosed, advanced ovarian cancer patients (pts) by BRCA1 or BRCA2 mutation in the phase III SOLO1 trial. Journal of Clinical Oncology. 2019;37(15_suppl):5551-.

4. Robson M, Im SA, Senkus E, Xu B, Domchek SM, Masuda N, et al. Olaparib for Metastatic Breast Cancer in Patients with a Germline BRCA Mutation. N Engl J Med. 2017;377(6):523-33.

5. Golan T, Hammel P, Reni M, Van Cutsem E, Macarulla T, Hall MJ, et al. Maintenance Olaparib for Germline BRCA-Mutated Metastatic Pancreatic Cancer. N Engl J Med. 2019;381(4):317-27.

6. Mateo J, Porta N, Bianchini D, McGovern U, Elliott T, Jones R, et al. Olaparib in patients with metastatic castration-resistant prostate cancer with DNA repair gene aberrations (TOPARP-B): a multicentre, open-label, randomised, phase 2 trial. Lancet Oncol. 2020;21(1):162-74.

7. Mateo J, Carreira S, Sandhu S, Miranda S, Mossop H, Perez-Lopez R, et al. DNA-Repair Defects and Olaparib in Metastatic Prostate Cancer. N Engl J Med. 2015;373(18):1697-708.

8. M. Hussain JM, K. Fizazi, F. Saad, N.D. Shore, S. Sandhu, K.N. Chi, O. Sartor, N. Agarwal, D. Olmos, A. Thiery-Vuillemin, P. Twardowski, N. Mehra, C. Goessl, J. Kang, J. Burgents, W. Wu, A. Kohlmann, C.A. Adelman, J. de Bono. PROFOUND: PHASE 3 STUDY OF OLAPARIB VERSUS ENZALUTAMIDE OR ABIRATERONE FOR METASTATIC CASTRATION-RESISTANT PROSTATE CANCER (MCRPC) WITH HOMOLOGOUS RECOMBINATION REPAIR (HRR) GENE ALTERATIONS. Annals of Oncology 2019;( 30 (suppl_5)):v851-v934.

9. Clarke N, Wiechno P, Alekseev B, Sala N, Jones R, Kocak I, et al. Olaparib combined with abiraterone in patients with metastatic castration-resistant prostate cancer: a randomised, double-blind, placebocontrolled, phase 2 trial. The Lancet Oncology. 2018;19(7):975-86.

10. Lavaud P, Gravis G, Foulon S, Joly F, Oudard S, Priou F, et al. Anticancer Activity and Tolerance of Treatments Received Beyond Progression in Men Treated Upfront with Androgen Deprivation Therapy With or Without Docetaxel for Metastatic Castration-na\&\#xef;ve Prostate Cancer in the GETUG-AFU 15 Phase 3 Trial. European Urology. 2018;73(5):696-703.

11. James ND, Sydes MR, Clarke NW, Mason MD, Dearnaley DP, Spears MR, et al. Addition of docetaxel, zoledronic acid, or both to first-line long-term hormone therapy in prostate cancer (STAMPEDE): survival results from an adaptive, multiarm, multistage, platform randomised controlled trial. The Lancet. 2016;387(10024):1163-77.

12. Kyriakopoulos CE, Chen YH, Carducci MA, Liu G, Jarrard DF, Hahn NM, et al. Chemohormonal Therapy in Metastatic Hormone-Sensitive Prostate Cancer: Long-Term Survival Analysis of the Randomized Phase III E3805 CHAARTED Trial. J Clin Oncol. 2018;36(11):1080-7.

13. Gravis G, Boher J-M, Chen Y-H, Liu G, Fizazi K, Carducci MA, et al. Burden of Metastatic Castrate Naive Prostate Cancer Patients, to Identify Men More Likely to Benefit from Early Docetaxel: Further Analyses of CHAARTED and GETUG-AFU15 Studies. European Urology. 2018;73(6):847-55. 
14. Armstrong AJ, Halabi S, Luo J, Nanus DM, Giannakakou P, Szmulewitz RZ, et al. Prospective Multicenter Validation of Androgen Receptor Splice Variant 7 and Hormone Therapy Resistance in High-Risk Castration-Resistant Prostate Cancer: The PROPHECY Study. J Clin Oncol. 2019;37(13):1120-9.

15. Scher HI, Lu D, Schreiber NA, Louw J, Graf RP, Vargas HA, et al. Association of AR-V7 on Circulating Tumor Cells as a Treatment-Specific Biomarker With Outcomes and Survival in Castration-Resistant Prostate Cancer. JAMA Oncology. 2016;2(11):1441-9.

16. Antonarakis ES, Lu C, Wang H, Luber B, Nakazawa M, Roeser JC, et al. AR-V7 and resistance to enzalutamide and abiraterone in prostate cancer. N Engl J Med. 2014;371(11):1028-38.

17. van der Toom EE, Axelrod HD, de la Rosette JJ, de Reijke TM, Pienta KJ, Valkenburg KC. Prostatespecific markers to identify rare prostate cancer cells in liquid biopsies. Nat Rev Urol. 2019;16(1):722.

18. Seitz AK, Thoene S, Bietenbeck A, Nawroth R, Tauber R, Thalgott M, et al. AR-V7 in Peripheral Whole Blood of Patients with Castration-resistant Prostate Cancer: Association with Treatment-specific Outcome Under Abiraterone and Enzalutamide. Eur Urol. 2017;72(5):828-34.

19. De Laere B, Rajan P, Gronberg H, Dirix L, Lindberg J, Core Arv CTC, et al. Androgen Receptor Burden and Poor Response to Abiraterone or Enzalutamide in TP53 Wild-Type Metastatic CastrationResistant Prostate Cancer. JAMA Oncol. 2019;5(7):1060-2.

20. Abida W, Campbell D, Patnaik A, Shapiro JD, Sautois B, Vogelzang NJ, et al. Non-BRCA DNA Damage Repair Gene Alterations and Response to the PARP Inhibitor Rucaparib in Metastatic CastrationResistant Prostate Cancer: analysis from the phase 2 TRITON2 study. Clinical cancer research : an official journal of the American Association for Cancer Research. 2020:clincanres.0394.2020.

21. Abida W, Bryce AH, Vogelzang NJ, Amato RJ, Percent I, Shapiro JD, et al. Preliminary results from TRITON2: A phase II study of rucaparib in patients (pts) with metastatic castration-resistant prostate cancer (mCRPC) associated with homologous recombination repair (HRR) gene alterations. Annals of oncology : official journal of the European Society for Medical Oncology. 2018;29 Suppl 8:viii272viii.

22. Niraparib Shrinks BRCA-Mutated Prostate Tumors. Cancer discovery. 2019;9(4):OF7-OF.

23. Clarke N, Wiechno P, Alekseev B, Sala N, Jones R, Kocak I, et al. Olaparib combined with abiraterone in patients with metastatic castration-resistant prostate cancer: a randomised, double-blind, placebocontrolled, phase 2 trial. Lancet Oncol. 2018;19(7):975-86.

24. Li L, Karanika S, Yang G, Wang J, Park S, Broom BM, et al. Androgen receptor inhibitor-induced "BRCAness" and PARP inhibition are synthetically lethal for castration-resistant prostate cancer. Science Signaling. 2017;10(480):eaam7479.

25. Xia B, Sheng Q, Nakanishi K, Ohashi A, Wu J, Christ N, et al. Control of BRCA2 cellular and clinical functions by a nuclear partner, PALB2. Mol Cell. 2006;22(6):719-29.

26. Goodall J, Mateo J, Yuan W, Mossop H, Porta N, Miranda S, et al. Circulating Cell-Free DNA to Guide Prostate Cancer Treatment with PARP Inhibition. Cancer Discov. 2017;7(9):1006-17. 
27. Gogola E, Rottenberg S, Jonkers J. Resistance to PARP Inhibitors: Lessons from Preclinical Models of BRCA-Associated Cancer. Annual Review of Cancer Biology. 2019;3(1):235-54.

28. Corn PG, Wang F, McKeehan WL, Navone N. Targeting fibroblast growth factor pathways in prostate cancer. Clin Cancer Res. 2013;19(21):5856-66.

29. Pilie PG, Tang C, Mills GB, Yap TA. State-of-the-art strategies for targeting the DNA damage response in cancer. Nat Rev Clin Oncol. 2019;16(2):81-104.

30. Yang X, Leslie G, Doroszuk A, Schneider S, Allen J, Decker B, et al. Cancer Risks Associated With Germline PALB2 Pathogenic Variants: An International Study of 524 Families. J Clin Oncol. 2020;38(7):674-85.

\section{Figures}

a.

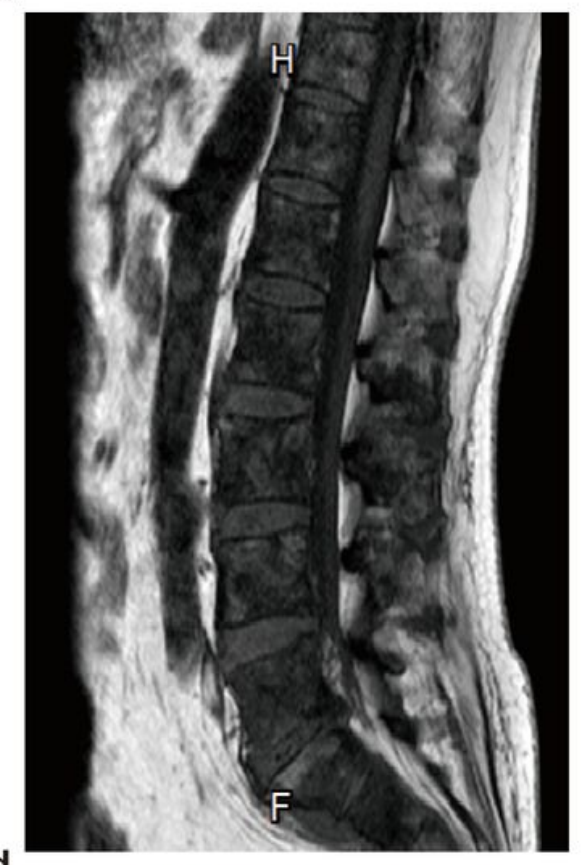

b.

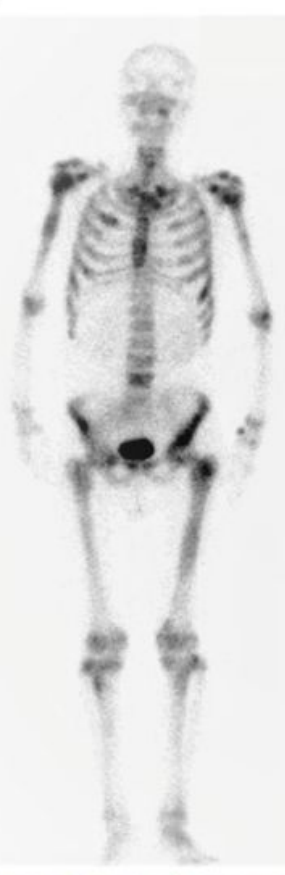

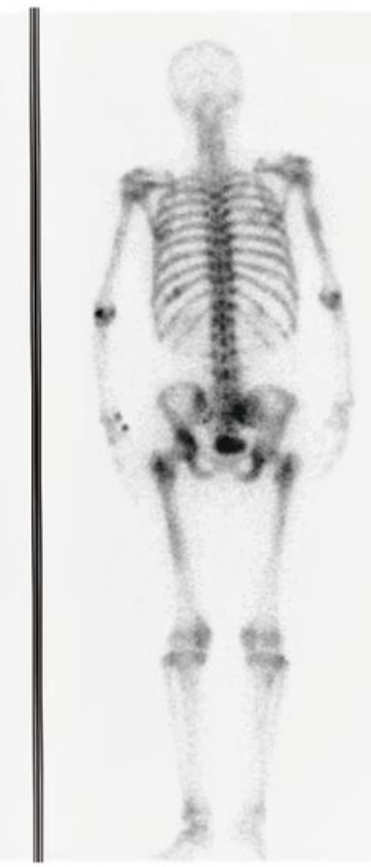

c.
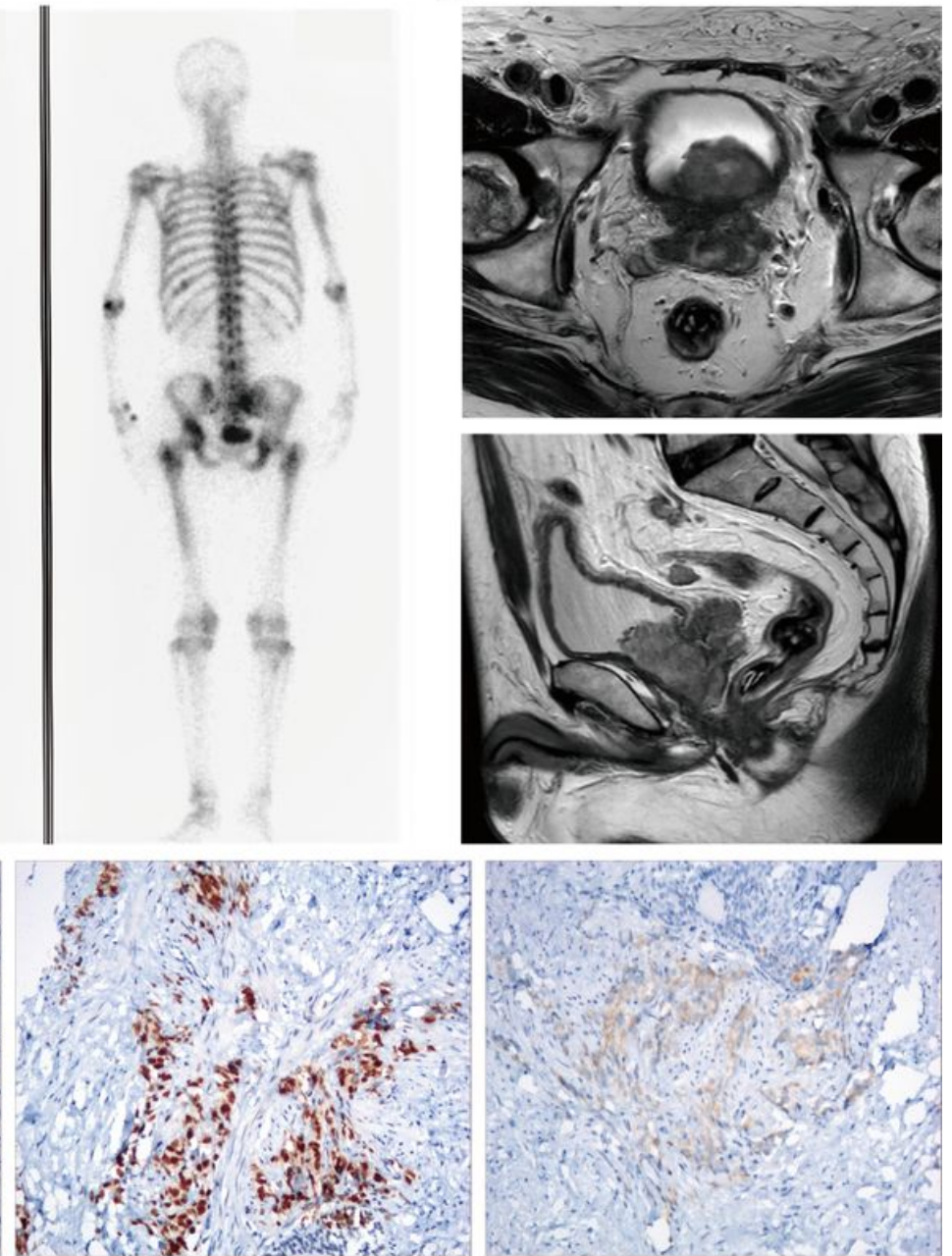

NKX3.1

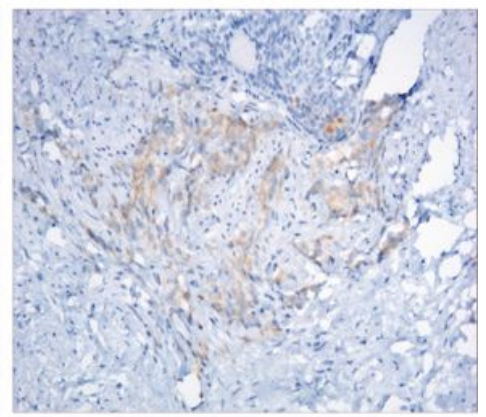

PSA

\section{Figure 1}

Radiological and immunohistochemical results showing metastatic prostate adenocarcinoma. a: Sagittal MRI showed multiple lumbar vertebrate metastasis. b: Bone scanning displayed highly metabolic activity of bone lesions. c: Cross (upper) and sagittal (lower) section of MRI scanning displayed bladder wall as 
well as seminal vesicle invasion of prostate malignant lesion. $\mathrm{d}$ : H\&E staining and immunohistochemistry resulted for AR, NKX3.1 and PSA staining.

a.

\begin{tabular}{|lccccc|}
\hline Circulating & /counts & $\begin{array}{c}\text { EMT-CTC } \\
\text { /counts }\end{array}$ & $\begin{array}{c}\text { M-CTC } \\
\text { /counts }\end{array}$ & $\begin{array}{c}\text { CTC clusters } \\
\text { / counts }\end{array}$ & $\begin{array}{c}\text { AR-V7 mRNA } \\
\text { expression }\end{array}$ \\
\hline 2017-06-13 & 1 & 6 & 0 & 0 & Negative \\
\hline 2018-06-19 $^{+}$ & 1 & 15 & 11 & 0 & Positive \\
\hline
\end{tabular}

b.
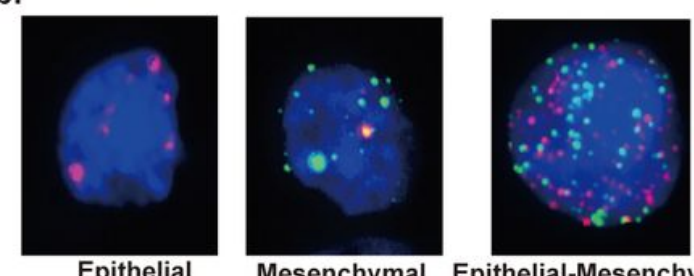

Mesenchymal Epithelial-Mesenchymal

c.

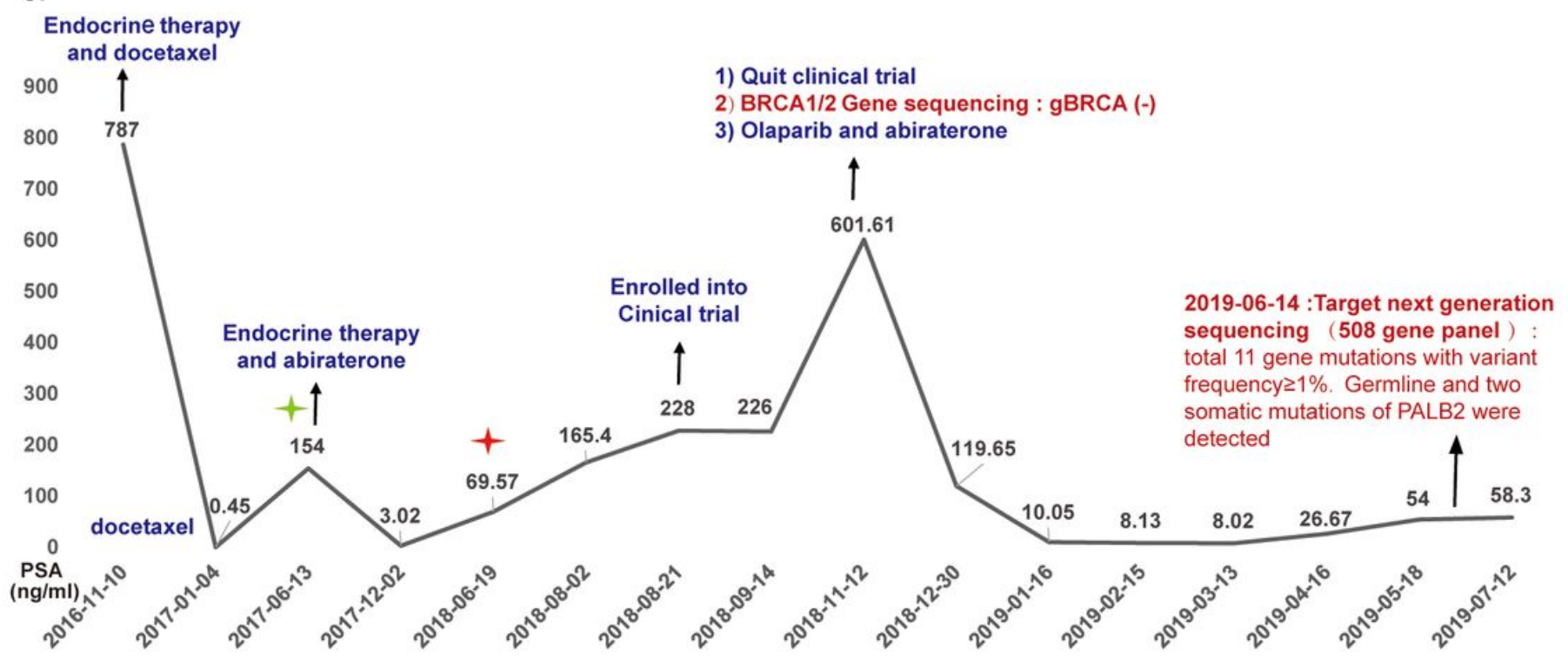

\section{Figure 2}

Results of CTCs detection and dynamic change of TPSA level. a: CTCs counts and classification change before and after abiraterone treatment, the expression of AR-V7 mRNA in these CTCs were detected. $b$ : Representative graphs displaying CTCs of three phenotypes. Red signal: Probe mixture detecting mRNA of EpCAM,CK8, CK18, CK19; Green signal: Probe mixture detecting mRNA of Vimentin and Twist. c: Dynamic change of TPSA level during overall treatment procedure with various therapeutic approaches. 


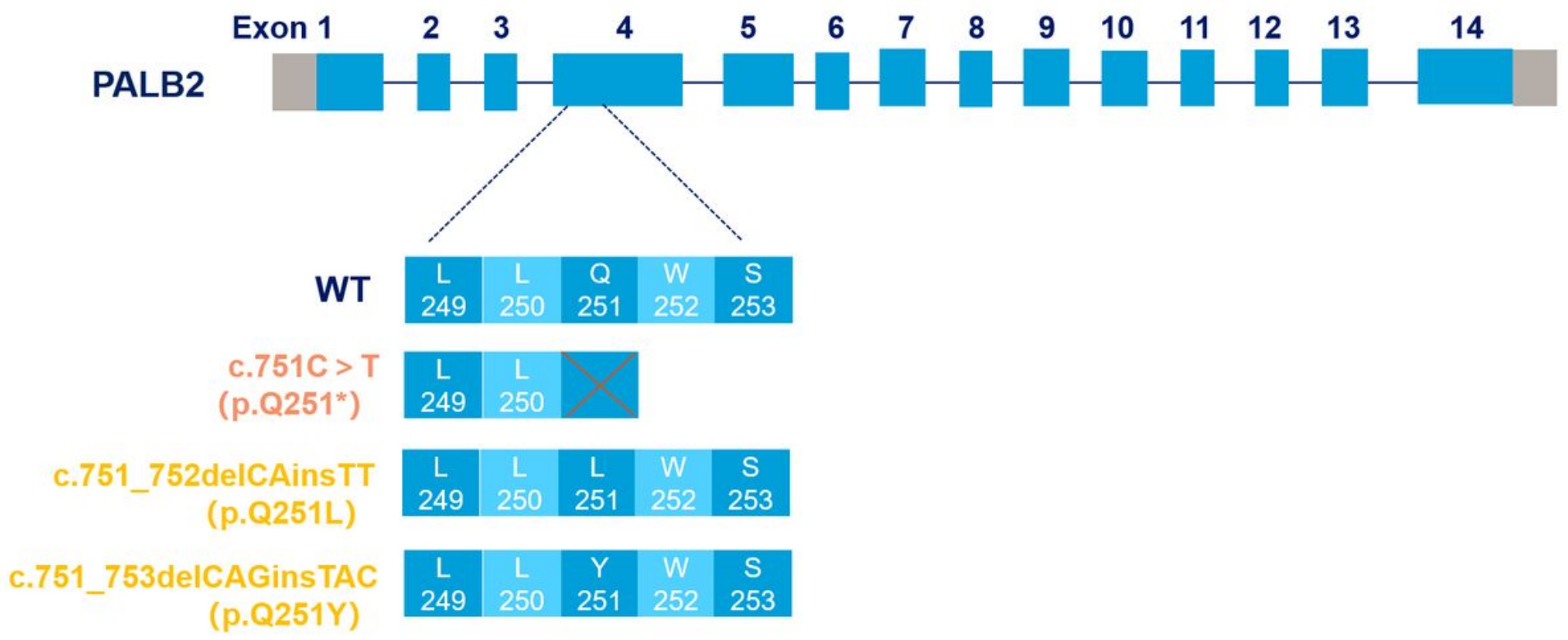

Figure 3

Diagram of PALB2 amino-acid sequence changes caused by the germlinemutation and two somatic mutations.

\section{Supplementary Files}

This is a list of supplementary files associated with this preprint. Click to download.

- Additionalfile1.docx

- CAREchecklistEnglish0703.pdf 